\title{
ILBEECP For Data Aggregation in Wireless Sensor Networks: A New Technique
}

\author{
Manpreet Kaur ${ }^{\mathrm{a}}$, Sandeep Kaur Dhanda ${ }^{\mathrm{b}}$ \\ ${ }^{a, b}$ Department of Computer Science and Engineering, BBSBEC Fatehgarh Sahib140407, Punjab, India
}

Received: 11 May 2018; Accepted: 09 August 2018; Published: 08 November 2018

\begin{abstract}
To design a load balanced energy efficient network has been a major concern in Wireless Sensor Networks (WSNs) since last many years. The load balancing energy-efficient clustering protocols are developed to attain the energy efficient network. But there was an issue related to the Cluster Head $(\mathrm{CH})$ selection process. As the selected $\mathrm{CH}$ has to transmit the data to the sink node whether the node is located at a farther distance and this leads to the extra consumption of the energy by the current $\mathrm{CH}$ node. In order to overcome this, the novel approach is developed which is based on the multilayer architecture. The proposed method elects the $\mathrm{CH}$ and then a Leader node is elected from the volunteer nodes to gather the data from respective $\mathrm{CH}$ and then to transmit it to the base station. The simulation results depict that ILBEECP (Improved Load balanced energy Efficient Clustering protocol) outperforms the LBEECP (Load balanced energy Efficient Clustering protocol) in the terms of the dead node, alive nodes, residual energy, and energy consumption.
\end{abstract}

Index Terms: Wireless Sensor Network, Energy Efficient Clustering Protocol, Load Balancing.

(C) 2018 Published by MECS Publisher. Selection and/or peer review under responsibility of the Research Association of Modern Education and Computer Science

\section{Introduction}

Wireless Sensor networks are made up by congregating a large number of tiny sensor devices. These sensor devices are conjoined to each other in order to transmit data from source to destination [1]. The deployed sensor operates on the basis of initially allotted energy. The sensor nodes communicate with each other by creating a route with the help of adjacent nodes. The short sensors having limited energy are generated by the current advancements in the technology that are limited in energy, computing power, and wireless communication capabilities. These sensor nodes can sense data after local processing transmit this data to the

* Corresponding author.

E-mail address:manpreetghangas@gmail.com 
base station directly or with the help of the forwarder node. The major demerit of these sensors is that the battery capacity of these sensors is very low. It is difficult to change or recharge these batteries. Therefore to improve the battery life of the sensor the data aggregation method is introduced. The main purpose of the data aggregation is to collect the data from several nodes by a single node and by applying aggregation it is transmitted to the base station. Due to this, the network lifetime is increased and the battery power is consumed very less. This process of path creation is known as routing [2]. In order to attain the effective routes, the concept of clustering comes to the existence. In clustering, the nodes with the similar nature are gathered into a single group and such multiple groups are created in the network, then a representative node is elected from each and every group and termed as Cluster Head $(\mathrm{CH})$ [3]. This $\mathrm{CH}$ is liable to transmit the data from cluster members to the sink node. By clustering, the energy efficiency capacity of the network can be enhanced. To make a network more energy efficient is the main consideration [4]. A large number of mechanism and technique have been developed to create an energy efficient network [5]. The load balancing is one that leads to an energy efficient wireless network [6]. Traditionally, to design a load balanced energy efficient network, simply the concept of clustering was applied which have not proved much beneficial.

This study develops an ILBEECP for load balancing energy efficient network. Rest of the work is organized to explain the problem in traditional LBCEEP protocol in Section II. Section III. Section IV. represents the proposed ILBCEEP protocol in brief along with its flow of working. Section V explains the results in a graphical form that is gathered from simulating the ILBEECP in MATLAB.

\section{Related Work}

A wireless sensor network faces several challenges in terms of load balancing. One of the major limitations of sensors is their low battery capacity. For some applications, there is no possibility of changing or recharging the battery of sensors. Hence, there is a need for an energy efficient protocol that saves energy by utilizing an appropriate strategy in intra-cluster and inter-cluster communications. This will result in load balancing and balanced energy consumption of the network. The research papers that were studied in sort to get the deep familiarity to increase Network Lifetime in Wireless Sensor Network is not new and has been discussed in the literature as follow:

Siavoshi et al.[1] had proposed an innovative clustering protocol of load balancing that alienated the entire network to the virtual circle with variable radiuses. The proposed protocol had used an innovative architecture in intracluster communication. In this protocol, if the distance from the base station was enlarged then the radius of all virtual cycles and also the size of all clusters would be enlarged in a way that the cluster size of all circles would be diverse as compared to the clusters of other circles. The head nodes were used to gather and constrict the information data from their regular neighbor nodes in all the clusters and transmit it to the head cluster. The simulation outputs had illustrated that the in the proposed protocol the network lifetime was enhanced compared to the conventional protocols. The energy effectiveness and load balancing were also offered in this protocol.

Wang et al.[3] had introduced a new hybrid multi-hop partition-based clustering (HMPBC) routing protocol. This proposed protocol could fit into a particular situation, interests and requirements were met, and the network lifetime of the system was also improved. In HMPBC, both the single-chain structure between the cluster and the cluster heads assortment on the basis of the residual energy were functioned by self-organization, and the region less spanning tree structure with clusters was recognized with the help of the base station. The simulation results of this protocol had demonstrated that the proposed HMPBC protocol had longer network lifetime, lesser variations in average energy consumption and zone parameters were accurately calculated comparative to the other conventional protocols such as LEACH-MLOR and the energy efficient hybrid multihop clustering scheme.

Xia et al.[10] present an energy-efficient and load-balanced dynamic clustering protocol based on artificial bee colony (EBABC) consist of two portions. The first portion had resolute the number of cluster heads on the basis of nodes' distribution and communication radiuses. The second portion was used to choose the cluster 
heads due to the residual energy, mobility, count of single-node cluster and distance of cluster heads from their neighbor nodes and to the server from cluster heads. Usual nodes used to link to the cluster head by in view of the cluster size and communication radius. The output had illustrated that the network lifetime of the protocol could effectively prolonged and had achieved load balance with the nodes.

Yaeghoobi et al.[16] had discussed an effectiveness of the routing protocols and the pros and cons of these routing protocols in several situations were also discussed in this paper. Moreover, the energy consumption, network lifetime and the packet transferring of these various protocols had been compared by entire performance evaluation and the simulations were examined. The simulation results had demonstrated that the energy efficiency of the DEEC protocol was better than the other conventional protocols with more alive nodes and least dead nodes. Additionally, in the heterogeneous and homogeneous environments, the performance of the HEED protocol was advanced than the SEP and LEACH protocols.

Tarunpreet et al.[17] had proposed a particle swarm optimization (PSO) based unequal and fault tolerant clustering protocol (PSO-UFC). This offered protocol had dealt with the imbalanced clustering and fault tolerance problems in the conventional energy-balanced unequal clustering (EBUC) protocol to achieve better or improved network lifetime of the system. The PSO-UFC protocol had employed an uneven clustering method to control intra-cluster and inter cluster energy consumption to resolve the imbalanced clustering trouble within the Master cluster heads (MCHs). Because of unexpected failure of $\mathrm{MCH}$ the network connectivity was restored with the help of choosing an additional $\mathrm{CH}$ known as Surrogate cluster head (SCH) in the proposed PSO-UFC protocol. The resultant output had illustrated that the network lifetime of the proposed PSO-UFC protocol was prolonged rather than the conventional protocols such as EBUC, PSO-C, and LEACH-C protocols.

Mohapatra et al.[18] had investigated the finest routing for more energy saving that was Load Balanced Energy Enhanced Clustered Bee Ad Hoc Routing (LBEE) with the protected PKI mechanism. The LBEE was motivated from the swarm intelligence and the bee colony algorithm had been tracked as the finest energy effective mechanism for the MANETs. In this study for the safety to be concerned with the energy effectiveness of each node of the network. The finest protection for the protocol had been selected as the four key protection methods.

\section{Problem Formation}

Due to the extensive increase in wireless communication, the energy load on the protocols has been increasing and to balance, this load over different protocols have become an important issue of consideration. Considering this fact, energy efficient clustering protocol was introduced which utilizes virtual circles that form a multilayer circular structure across the network and make use of an appropriate strategy in intra-cluster communications that caused load balancing and balanced distribution of energy consumption around the network. This strategy included a downside of selecting a cluster head. The selection of the cluster head is performed on the basis of a single parameter i.e. distance. The cluster head is a node that is responsible for sending as well as receiving data packets from different leader nodes to the base station. Therefore, it must be filled with a sufficient amount of energy so that it can receive and send the packets to the base station without facing any obstacles. Consequently, distance should not be the only parameter for the election of a cluster head. Additionally, in the existing technique, leader nodes of a particular region forward their data packets to the cluster head only. Sometimes, the leader nodes have to travel great distance that enhanced the consumption of energy. Therefore, a novel approach should be proposed that reduced overall traveled distance of leader nodes while electing appropriate cluster head of a region. The main objectives of the proposed work is described below:

- To study the various energy efficient clustering protocol in WSN.

- To develop an algorithm for electing the cluster head on the basis of the enhanced number of parameters.

- To establish inter-region communication between leader node and cluster head in a particular region. 
- To compare and evaluate the proposed energy efficient clustering protocol with the existing protocol.

\section{Proposed Work}

In the existing energy-efficient clustering protocol, cluster head has been elected on the basis of distance parameter. As a cluster head requires a high amount of energy for both receiving and sending of data packets and to balance such load on it, so a single parameter is not enough for their election. In the proposed work, distance is another parameter which considered with the energy parameter for the selection of a cluster head in a region. The leader nodes in the region forward their data packets to the chosen cluster head. Another problem in the existing technique was the total distance leader nodes have to cover for sending of packets. To overcome this problem, the concept of inter-region communication has introduced. In this aspect, a distance of leader nodes with the cluster heads and other leader nodes is evaluated. From the evaluation, the node with least distance either from cluster head or another leader node will be selected for forwarding of data packets. The implementation of this concept will minimize the consumption of energy and reduce the load on the cluster head. Moreover, the lifetime of the network will also be improved while enhancing the efficiency of the proposed technique. The methodology of proposed work is as follows:

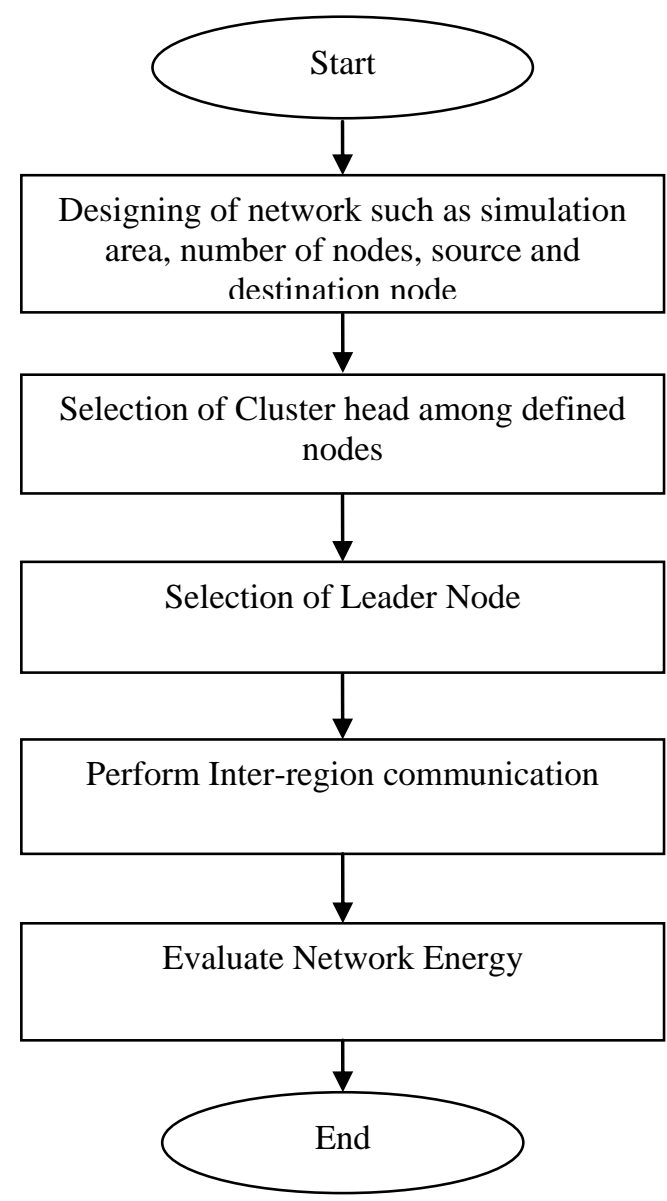

Fig.1. Flow of Proposed Work 
The stepwise procedure of proposed work is defined as follows:

a) Start

b) Initialize or design the network by initializing the various parameters such as the area of the network, a number of nodes in the network, location for nodes deployment in the network, amount of initial energy of the nodes, sink node and source node.

c) The network is arranged in multilayer architecture. Next step is to select the $\mathrm{CH}$ from the deployed nodes.

d) In this step, the leader node is selected from the volunteer nodes.

e) To transmit the data by using Leader Node. It is the responsibility of Leader Node to collect the data from $\mathrm{CH}$ and then transmit this data to the Sink node. After performing the data transmission, the last step is to evaluate the performance of the network by measuring various parameters such as a number of dead nodes, alive nodes, energy consumption, residual energy etc.

\section{Experiment and Analysis}

The ILBEECP aims to enhance the network lifetime by reducing the energy consumption by the nodes in a network. To achieve so, the concept of multilayer architecture is implemented. Along with this, the Leader node is also selected among the volunteer nodes in each layer of the network. The detail of simulation parameters for ILBEECP is given in the table below:

Table 1. Simulation Parameters

\begin{tabular}{cc}
\hline Parameters & Value \\
\hline Area of Network & $300 \times 300$ \\
Number of Nodes & 504 \\
Location of Sink Node & $150 \times 150$ \\
Initial Energy & 0.5 joule \\
Free Space Channel Model & $50 \mathrm{~nJ} / \mathrm{bit}$ \\
Multi-Path Fading Channel Model & $0.013 \mathrm{pJ} / \mathrm{bit} / \mathrm{signal}$ \\
Distance Threshold & $87 \mathrm{~m}$ \\
Data Aggregation Energy & $5 \mathrm{~nJ} / \mathrm{bits} / \mathrm{signal}$ \\
Data Packet Size & 4000 \\
Control Packet Size & 200 \\
\hline
\end{tabular}

This section organizes the results that are obtained after implementing the proposed work in MATLAB. The results are evaluated in the terms of dead nodes, alive nodes, remaining energy, and energy consumption.

The graph in figure 2 represents the structure of the network given in the proposed work. In the proposed work, the multilayer network structure is followed. The graph depicts that total three types of nodes are considered for creating multilayer architecture. These nodes are Cluster Members, Cluster Heads, and Leader nodes. The nodes are in green color represents the volunteer nodes for leader node, nodes in black color represents the cluster member nodes and nodes in red depicts the Cluster Head Node. The sink node is shown 
in green color and is located at the center of the network. The area of the network is $300 * 300$ and the number of nodes in the network is 504 .

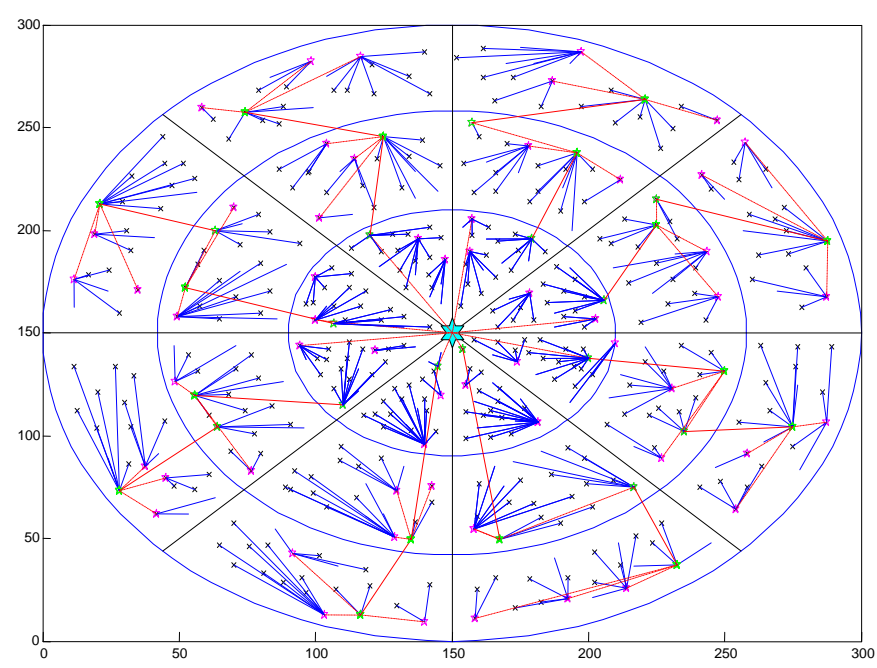

Fig.2. Proposed Network Structure

The graph in figure 3 delineates the number of dead nodes in the proposed network. The dead node refers to the nodes in the network which do not have sufficient amount of energy to perform data transmission in the network. The number of dead nodes in the network should be low. If this number reaches to higher then this can have worse effects on the performance of the network. The number of dead nodes in the proposed work is evaluated on the basis of the number of communication rounds in the network. Total 2000 rounds of communication are considered in proposed work. Initially, the number of dead nodes in the network is 0 and it remains 0 until the completion of 1300 rounds. The number of dead nodes in proposed work is measured to be 146 at 1301 round.

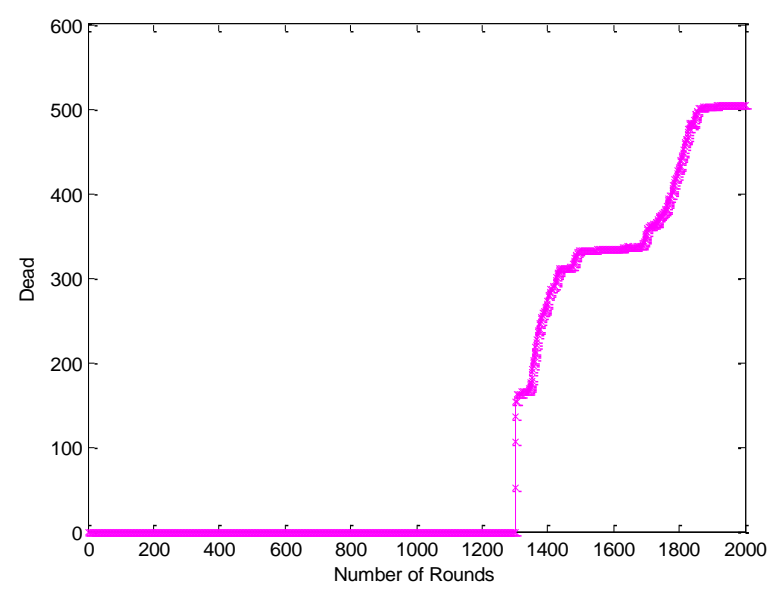

Fig.3. Dead Nodes in Proposed Network 
The alive nodes refer to the nodes in the network that are capable enough to run the network. Figure 4 shows the graph of a number of alive nodes in the proposed network. The x-axis in the graph depicts the number of communication rounds; the y-axis shows the number of alive nodes in the network. Initially, total 504 nodes are in alive mode but this count started falling at around 1300 and till the completion of rounds 0 nodes are found alive.

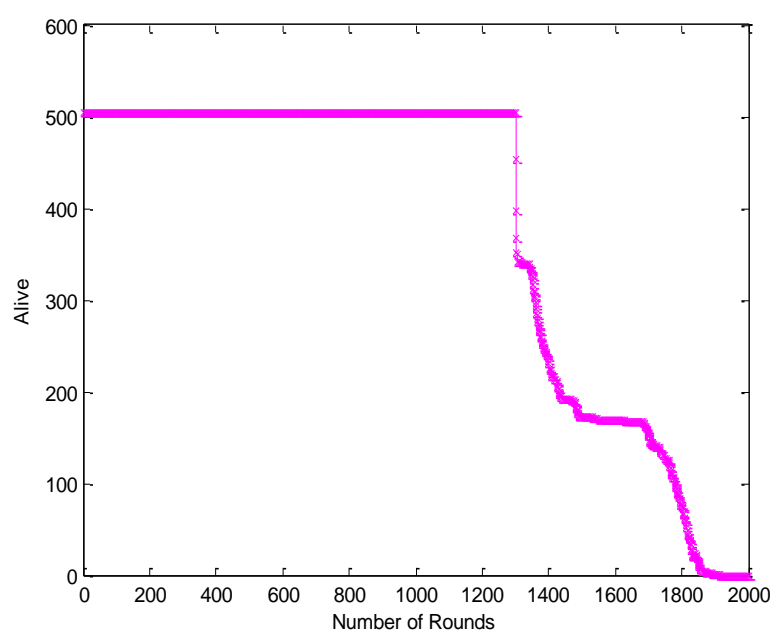

Fig.4. Alive Nodes in Proposed Work

Figure 5 represents the graph for showing the remaining energy of the nodes in the proposed work. The remaining energy or residual energy is also measured with respect to the number of communication rounds. The y-axis represents the amount of remaining energy and it is measured in joule. The graph explains that the energy of nodes is higher when the number of communication rounds is lower but the amount of energy is getting reduced as the number of rounds in the communication is increasing.

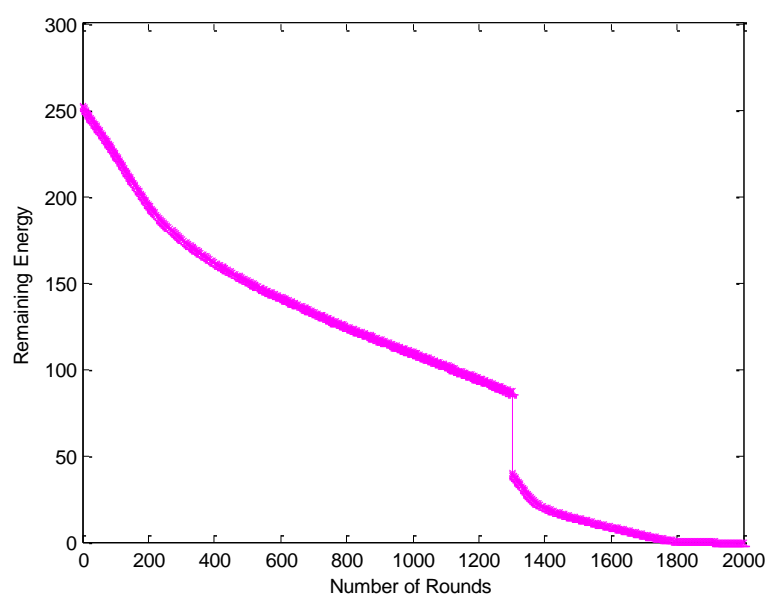

Fig.5. Remaining energy of the nodes in proposed work 
Figure 6 shows the energy consumption level of the nodes in the proposed work. The graph makes it clear that when the number of rounds is 0 then the energy consumption is also 0 . As the number of rounds is getting increased, the nodes have to perform data transmission from $\mathrm{CH}$ to sink. Thus the nodes consume energy to perform the task of transmission. It is observed from the graph that the till the completion of the network, the energy consumption level reaches to the highest but this is achieved after completion of communication rounds.

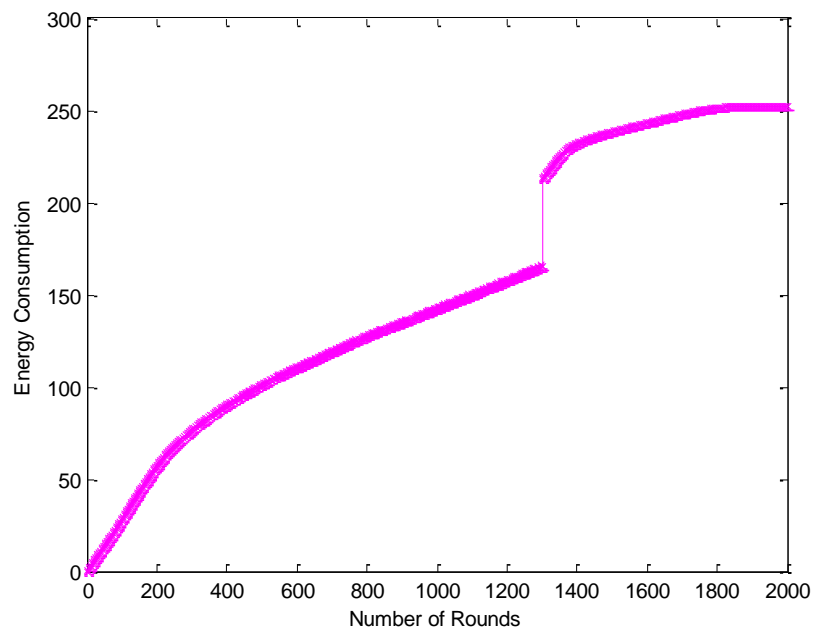

Fig.6. Energy consumption in proposed work

The comparison of proposed i.e. Improved LBEECP (ILBEECP) and traditional LBEECP is depicted in figure 7. The comparison is drawn on the basis of the total remaining energy of the network. The curve in magenta color depicts the results for ILBEECP and curve in blue color shows the results for LBEECP. The remaining energy of the LBEECP gets exhausted after completion of 1058 rounds whereas for ILBEECP the nodes get exhausted at 1868 round. Thus it is observed that the proposed work is more efficient than the traditional work with respect to the total remaining energy of the nodes

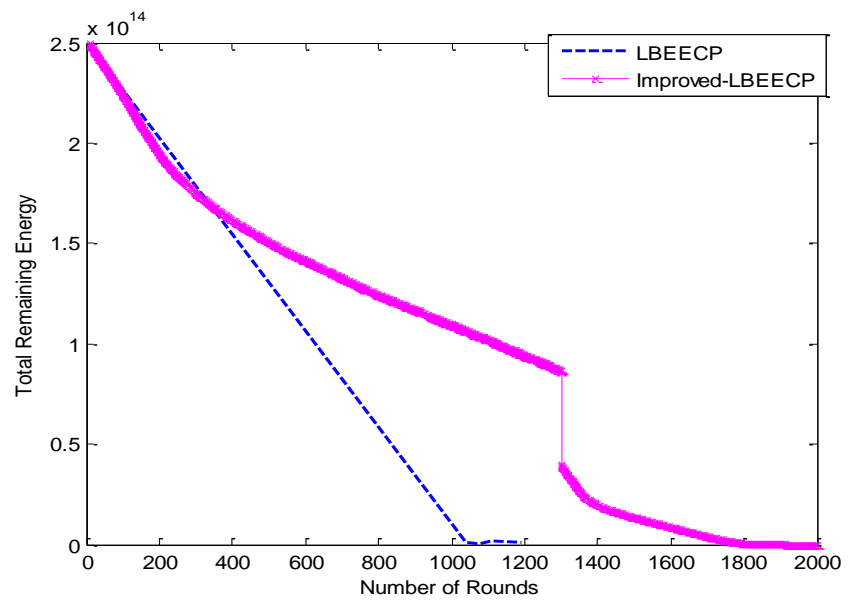

Fig.7. Comparison of remaining energy 
Table 2 depicts the comparison of remaining energy between LBEECP and I-LBEECP.

Table 2.Comparison of Remaining Energy

\begin{tabular}{ccc}
\hline Rounds & I-LBEECP & LBEECP \\
\hline 300 & $2.436 \mathrm{e}+014$ & $2.436 \mathrm{e}+014$ \\
1038 & $1.069 \mathrm{e}+014$ & $1.069 \mathrm{e}+014$ \\
1193 & $9.505 \mathrm{e}+013$ & $1.069 \mathrm{e}+014$ \\
\hline
\end{tabular}

Figure 8 defines the comparison of a number of alive nodes in LBEECP and ILBEECP. The observations explain that the number of alive nodes in both cases is the same. In the case of LBEECP, the count of LBEECP started falling at 995 round of communication. Whereas in ILBEECP the number of alive nodes stared decreasing at round 1300. It is mandatory that the number of alive nodes should be high in the network and from the observations it is proved that the proposed work has a higher number of alive nodes in comparison to the LBEECP protocol.

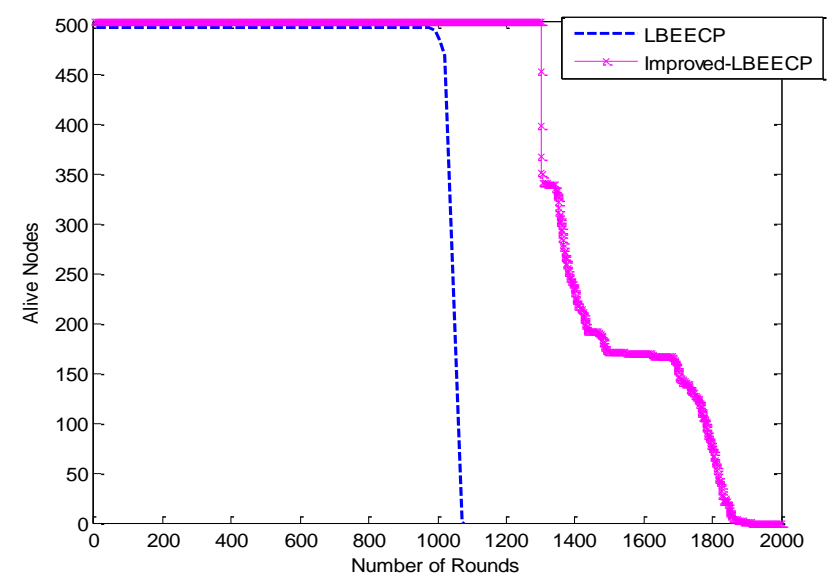

Fig.8. Comparisons of Alive Nodes

Table 3 depicts the comparison of alive nodes in traditional and proposed work with respect to the different number of rounds.

Table 3. Comparison of Alive Nodes

\begin{tabular}{ccc}
\hline Rounds & I-LBEECP & LBEECP \\
\hline 200 & 504 & 504 \\
800 & 504 & 504 \\
1200 & 504 & 0 \\
1600 & 173 & 0 \\
20000 & 3 & 0 \\
\hline
\end{tabular}


The graph in figure 9 represents that the number of dead nodes in ILBEECP is lower in comparison to the LBEECP. Initially, the number of dead nodes is found to be 0 in both cases. In LBEECP, the number of dead nodes reaches to the 500 at the end of 1042 rounds whereas in ILBEECP the number of dead nodes at the same communication round is 0 . The proposed work has better results in terms of dead node in comparison to the LBEECP.

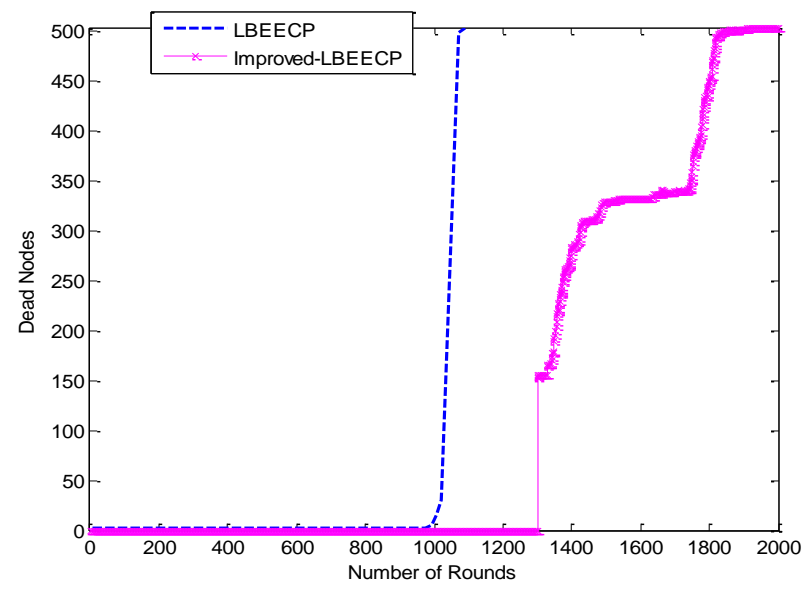

Fig.9. Comparisons of Dead Nodes

Table 4 represents the comparison of dead nodes in LBEECP and I-LBEECP at a various number of rounds.

Table 4. Comparison of Dead Nodes

\begin{tabular}{ccc}
\hline Rounds & I-LBEECP & LBEECP \\
\hline 200 & 0 & 0 \\
800 & 0 & 0 \\
1200 & 0 & 504 \\
1600 & 331 & 504 \\
20000 & 504 & 504 \\
\hline
\end{tabular}

\section{Conclusions \& Future Scope}

The work concentrates on the importance of load balanced energy efficiency in a Wireless Sensor Networks. It is observed that the traditional LBEECP mechanism was not much effective thus ILBEECP is developed to overcome its shortcomings. The ILBEECP elects the $\mathrm{CH}$ on the basis of energy and distance of the nodes. Along with this, the leader node is elected for representing the $\mathrm{CHs}$ in the process of communication. On the basis of the results, it is observed that the ILBEECP is performed outstandingly in comparison to LBCEEP. In the future, more advanced intelligence based optimization technique can be used for improving the results. 


\section{Acknowledgment}

The authors would like to thank anonymous reviewers for their careful reading of this paper and for their helpful comments.

\section{References}

[1] Saman Siavoshi, Yousef S. Kavian, Hamid Sharif, "Load-balanced energy efficient clustering protocol for wireless sensor networks", IET, Vol 6, Issue 3, Pp 67-73, 2016.

[2] Dali Wei, Yichao Jin, Serdar Vural, Klaus Moessner, Rahim Tafazolli, “An Energy-Efficient Clustering Solution for Wireless Sensor Networks”, IEEE, Vol 10, Issue 11, Pp 3973 - 3983, 2011.

[3] Charming Wang, Yuan Zhang, Xuewen Wang, Zhiyong Zhang, "Hybrid Multihop Partition-Based Clustering Routing Protocol for WSNs", Vol 2, Issue 1, 2018.

[4] Xiaomin Li, Di Li, Song Li, Shiyong Wang, Chengliang Liu, "Exploiting Industrial Big Data Strategy for Load Balancing in Industrial Wireless Mobile Networks", IEEE, Vol 6, Pp 6644 - 6653, 2017.

[5] Damodar Reddy Edla, Amruta Lipare, Ramalingaswamy Cheruku, Venkatanareshbabu Kuppili, "An Efficient Load Balancing of Gateways Using Improved Shuffled Frog Leaping Algorithm and Novel Fitness Function for WSNs", Vol 17, Issue 20, Pp 6724 - 6733, 2017.

[6] Peyman Neamatollahi, Mahmoud Naghibzadeh, Saeid Abrishami, "Fuzzy-Based Clustering-Task Scheduling for Lifetime Enhancement in Wireless Sensor Networks”, IEEE, Vol 17, Issue 20, Pp 6837 6844, 2017.

[7] Ray, A., De, D., "Energy efficient clustering protocol based on K-means(EECPK-mean) midpoint algorithm for enhanced network lifetime in wireless sensor network", IET Wireless Sensor System, Vol. 6, No. 6,Pp 181-191,2016

[8] Salim, A., Osamy, and W., Khedr, A. M.,"IBLEACH: intra-balanced LEACH protocol for wireless sensor networks", Wireless Networks, Vol. 20, Pp.1515-1525, 2014

[9] Nayebi, A., and Sarbazi-Azad, H., "Performance modeling of the LEACH protocol for mobile wireless sensor networks", Journal of parallel and distributed computing, Vol. 71, No. 6, Pp. 812-821, 2011.

[10] Xia, F., Zhao, X., Liu, H., Li, J. and Kong, X., “ An energy-efficient and load-balanced dynamic clustering protocol for ad-hoc sensor networks", Cyber Technology in Automation, Control, and Intelligent Systems (CYBER), 2012 IEEE International Conference on (pp. 215-220). IEEE,2012

[11] A.Tarachand, Vikas Kumar, Abhishek Raj, Ashish Kumar, Prasanta K. Jana, "An Energy-efficient Load Balancing Algorithm for cluster-based wireless sensor networks", IEEE, 2013.

[12] Feng Xia, Xuhai Zhao, Haifeng Liu, Jing Li, Xiangjie Kong, "An energy-efficient and load-balanced dynamic clustering protocol for ad-hoc sensor networks", IEEE, 2012.

[13] Anirooth Thonklin, W. Suntiamorntut, "Load balanced and energy efficient cluster head election in Wireless Sensor Networks", IEEE, 2011.

[14] Jyoti Singh, "A New LEACH-based Routing Protocol for Energy Optimization in Wireless Sensor Network” IEEE, Pp 181-186, 2014.

[15] Amit Sharma and Parul Kansal, "Energy efficient load-balanced clustering algorithm for Wireless Sensor Network", India Conference (INDICON), 2015 Annual IEEE, 2015.

[16] S.B.Kaebeh Yaeghoobi; M.K.Soni; S.S.Tyagi, "Performance of energy efficient clustering protocols to maximize Wireless Sensor Networks Lifetime “, ICSCTI, Pp 170-176,2015.

[17] Tarunpreet Kaur; Dilip Kumar, "Particle Swarm Optimization based Unequal and Fault Tolerant Clustering Protocol for Wireless Sensor Networks", IEEE Sensors Journal, Pp 1-1, 2018.

[18] Sasmita Mohapatra; M. Siddappa, "Enhancing security for load balanced energy enhanced clustered bee ad-hoc network using secret public keys", ICIMIA, Pp 343 - 348, 2017. 


\section{Authors' Profiles}

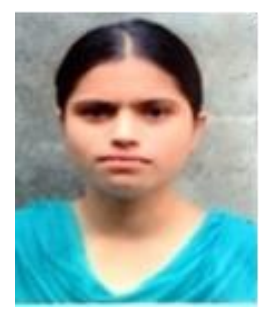

Manpreet Kaur, received her B.Tech degree in Computer Science \& Engineering from Guru Nanak Dev Engineering College, Ludhiana, Punjab, India and her Master`s degree in Computer Science \& Engineering from Baba Banda Singh Bahadur Engineering College, Fatehgarh Sahib, Punjab, India. Her current research interests include Load Balancing, Clustering and Network stability in Wireless Sensor Networks.

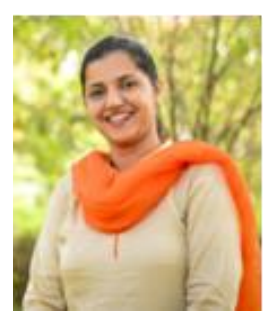

Sandeep Kaur Dhanda, received her Master`s degree in Computer Science \& Engineering from Thapar University, Patiala, Punjab, India. Presently, She is an assistant professor at Computer Science \& Engineering Department of Baba Banda Singh Bahadur Engineering College, Fatehgarh Sahib, India. Her research area includes Database, Wireless Communications and Parallel Computing. She is available at sandeep.tiwana@bbsbec.ac.in

How to cite this paper: Manpreet Kaur, Sandeep Kaur Dhanda, "ILBEECP For Data Aggregation in Wireless Sensor Networks: A New Technique", International Journal of Wireless and Microwave Technologies(IJWMT), Vol.8, No.6, pp. 50-61, 2018.DOI: 10.5815/ijwmt.2018.06.04 